\title{
Norois
}

Environnement, aménagement, société

$210 \mid 2009 / 1$

L'agriculture et les filières agro-alimentaires en France et en Argentine

\section{Évolutions récentes des filières du sucre et du citron de Tucumán (Argentine)}

Present developments of sugar and lemon agrobusiness in Tucuman, Argentina Los complejos agro-industriales del azúcar y del limón en la provincia de

Tucumán

\section{Elena del Valle Cueto}

\section{(2) OpenEdition}

Journals

Édition électronique

URL : https://journals.openedition.org/norois/2806

DOI : $10.4000 /$ norois.2806

ISBN : 978-2-7535-1558-1

ISSN : $1760-8546$

\section{Éditeur}

Presses universitaires de Rennes

\section{Édition imprimée}

Date de publication : 1 mars 2009

Pagination : 81-90

ISBN : 978-2-7535-0844-6

ISSN : 0029-182X

\section{Référence électronique}

Elena del Valle Cueto, «Évolutions récentes des filières du sucre et du citron de Tucumán (Argentine) », Norois [En ligne], 210 | 2009/1, mis en ligne le 01 mars 2011, consulté le 14 janvier 2022. URL : http://journals.openedition.org/norois/2806 ; DOI : https://doi.org/10.4000/norois.2806

Ce document a été généré automatiquement le 14 janvier 2022.

(c) Tous droits réservés 


\section{Évolutions récentes des filières du sucre et du citron de Tucumán (Argentine)}

Present developments of sugar and lemon agrobusiness in Tucuman, Argentina Los complejos agro-industriales del azúcar y del limón en la provincia de Tucumán

Elena del Valle Cueto

\section{NOTE DE L'ÉDITEUR}

Cet article a été reçu le 19 juillet 2008 et définitivement accepté le 10 mars 2009.

1 Une série de changements agricoles se sont produits dans le Nord-Ouest argentin, avec d'importantes conséquences au niveau territorial, économique et social, particulièrement dans la province de Tucumán. L'existence du NOA (Nord-Ouest argentin) est fondée sur des régions historiques, au-delà des formes d'organisation et d'administration qui lui ont succédé et malgré une grande diversité des environnements naturels. Le Nord-Ouest argentin est situé dans la région andine centrale de l'Amérique du Sud. Il occupe $17 \%$ de la superficie continentale et regroupe $11 \%$ de la population totale du pays. Il comprend les provinces de Salta, Jujuy, Santiago del Estero, Catamarca et Tucumán. Il s'agit d'un territoire d'une grande complexité physique (fig. 1), aux variations climatiques assez remarquables. Les cordons montagneux constituent des barrières physiques qui arrêtent les vents, provoquent des pluies qui alimentent les bassins hydrographiques, déterminent un étagement climatique qui provoque un étagement des formations végétales. Ce territoire peut être défini par deux éléments morphostructuraux de superficie presque identique : le système montagneux vers l'ouest et la plaine chaqueña vers l'est. La région présente de grandes variations bioclimatiques, depuis le sous-climat aride andin de la " puna », la zone aride des montagnes et des dépressions, le climat tropical montagnard, jusqu'au 
climat tropical à saison sèche, résultat de la combinaison des différentes altitudes, latitudes et hauteurs des reliefs montagneux qui le définissent. Cet environnement physique conditionne fortement l'occupation agricole du territoire (fig. 2).

Figure 1 : Le découpage administratif du Nord-Ouest argentin Administration pattern of the North Western part of Argentina

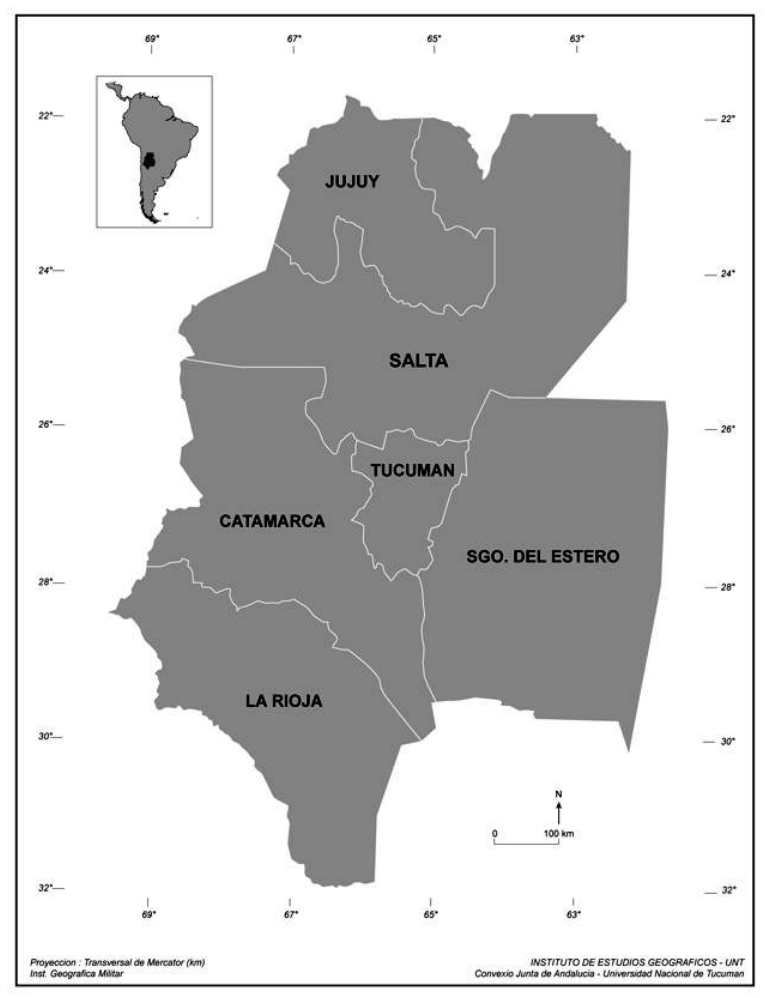

2 La région possède une identité propre, elle se reconnaît d'elle-même et préexiste à la Nation. Jusqu'au milieu du XVIII siècle, le NOA a été l'une des régions économiques les plus dynamiques et les plus peuplées de ce qui, plus tard, est devenu la République Argentine. Son activité économique se basait sur le commerce avec le centre minier de Potosí au Pérou. En raison des changements politiques du début du XIX siècle, BuenosAires et la région pampeana se sont intégrées à l'économie mondiale dans le cadre d'une politique de libre-échange. Le reste du pays, dont le NOA, a été laissé en marge des circuits commerciaux externes. Dans ce contexte, la province de Tucumán est devenue le principal centre socio-économique du NOA, en s'intégrant au contexte économique national grâce à l'agro-industrie du sucre, destiné au marché interne.

Régionalement, la production sucrière s'organise selon deux systèmes différents et parfois antagonistes. Dans les secteurs de Salta et de Jujuy, les raffineries de sucre travaillent avec de grands domaines qui leur garantissent l'approvisionnement en matières premières et favorisent la diminution des coûts de production. Par ailleurs, n'étant pas un espace soumis à la monoculture, une certaine diversification agricole et industrielle permet à cette petite région de surmonter les crises sucrières. Dans le secteur de Tucumán en revanche, 60 \% de la superficie cultivée correspond à la canne à sucre, ce qui détermine une partie très importante du produit intérieur brut de la province. L'activité du citron est plus récente dans la région et notamment à Tucumán. C'est dans les années 1960 que deux faits historiques ont déterminé l'expansion de la production de citron à Tucumán : d'une part, une grande crise au sein de l'industrie 
sucrière orchestrée par le gouvernement du Président Onganía (1966-1970)1, et d'autre part, une perte des plantations d'oranges qui a obligé les producteurs à s'orienter vers la production du citron (Batista et Natera Rivas, 2001).

4 L'évolution de ce système sucrier de Tucumán et de la diversification vers la production de citrons à partir des années 1960, fournit un très bon exemple des restructurations de la filière agro-industrielle ${ }^{2}$ dans la province de Tucuman. Les aspects agricoles et commerciaux servent de repères pour mesurer les articulations entre territoires, producteurs, acteurs sociaux et économiques, entre les circuits de production et les industries agro-alimentaires, entre l'agro-industrie régionale et les exigences $\mathrm{du}$ marché intérieur et mondial.

Figure 2 : Carte physique du Nord-Ouest argentin (Source : EEAOC) Argentinian NW Physical map

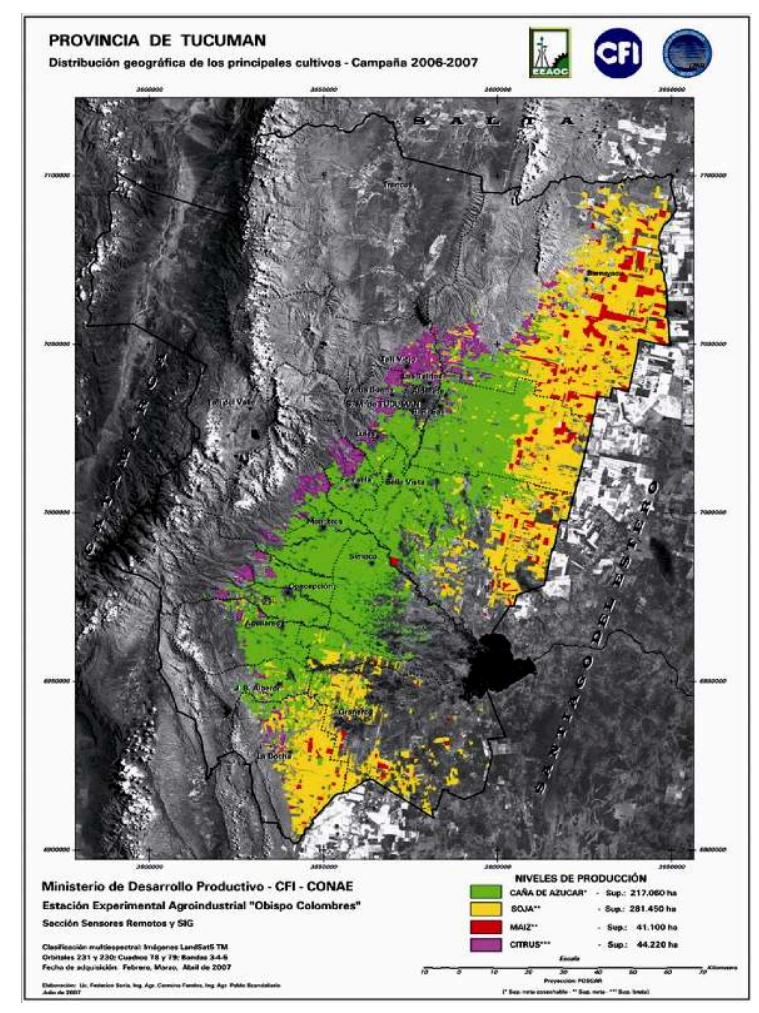

\section{Le circuit productif du sucre}

5 L'aire de la canne à sucre occupe la plaine centrale de la province de Tucumán, connue sous le nom de plaine " chaco-pampeana ", et dont les précipitations annuelles se situent entre 1000 et $750 \mathrm{~mm}$. Cette zone de culture s'est étendue peu à peu vers l'ouest sur le piémont, et vers le sud de la province. Les gelées et la saturation en eau du sol constituent un facteur limitant le développement de la canne à sucre, alors que l'amplitude thermique quotidienne aide à son mûrissement. En 2006, dans la province de Tucuman, 203000 hectares plantés ont donné un rendement à l'hectare de 69,2 tonnes de canne, soit 8,2 tonnes de sucre par hectare (tableau 1). $70 \%$ de la production de canne à sucre sont issus d'agriculteurs indépendants (les exploitations moyennes et grandes produisent $80 \%$ des volumes et les petites exploitations de moins de 10 ha produisent $20 \%$ ). Sur la totalité de la canne moulue, $8,2 \%$ correspondent à la canne à 
sucre que possèdent directement les raffineries, et 91,8 \% à celle des producteurs indépendants.

Tableau 1 : La production sucrière de la province de Tucuman de 1990 à 2007 Sugar production in Tucuman between 1990 and 2007

\begin{tabular}{|c|c|c|c|c|c|}
\hline Récolte & $\begin{array}{c}\text { Surface cultivée } \\
\text { (ha) }\end{array}$ & $\begin{array}{c}\text { Sucre } \\
\text { (tonnes) }\end{array}$ & Canne moulue $(\mathrm{t})$ & $\begin{array}{c}\text { Rendement } \\
\text { (t/ha) }\end{array}$ & RendementManufacturier (\%) \\
\hline 1990 & 250000 & 611322 & 6654578 & 38,68 & 9,2 \\
\hline 1991 & 250000 & 877242 & 8788611 & 42,34 & 10 \\
\hline 1992 & 250000 & 756840 & 8019099 & 43,26 & 9,4 \\
\hline 1993 & 224800 & 560550 & 6132255 & 38,85 & 9,3 \\
\hline 1994 & 224800 & 640413 & 6912664 & 40,11 & 10,4 \\
\hline 1995 & 225100 & 920157 & 8833895 & 46,49 & 8,8 \\
\hline 1996 & 210000 & 793419 & 8976865 & 53,97 & 10,5 \\
\hline 1997 & 202000 & 1049734 & 9948.502 & 52,27 & 9,1 \\
\hline 1998 & 222000 & 1153248 & 11328749 & 67,02 & 9,3 \\
\hline 1999 & 202700 & 956785 & 10274749 & 52,12 & 9,4 \\
\hline 2000 & 184100 & 892396 & 9594595 & 51,32 & 10,2 \\
\hline 2001 & 183390 & 862709 & 9145226 & 52,21 & 10,3 \\
\hline 2002 & 185720 & 914341 & 8987049 & 53,41 & 9,8 \\
\hline 2003 & 188920 & 1094736 & 10568847 & 51,16 & 11,1 \\
\hline 2004 & 200530 & 1030352 & 10518534 & 52,17 & 11,2 \\
\hline$* 2005$ & 193120 & 1267288 & 11450965 & 63,00 & 9,6 \\
\hline$* 2006$ & 203170 & 1524880 & 13602593 & 69,39 & \\
\hline$* 2007$ & 217060 & 1279133 & 13324735 & & \\
\hline
\end{tabular}

* chiffres provisoires

Canne à sucre moulue, rendement manufacturier \%, et sucre produit 1990-2007 CAA

\section{La phase agroindustrielle}

6 À partir de 1876, l'arrivée du chemin de fer et l'industrialisation de la canne à sucre installèrent dans la province, un ordre économique capitaliste (Bravo, 1993, cité par Rozenzwaig, 1997) et instaurèrent un nouveau système de relations interrégionales. La modernisation de l'industrie sucrière a généré des acteurs sociaux de trois types. D'une part, les industriels ont acquis grâce à l'industrie sucrière, un pouvoir politique provincial, articulé au niveau national (ils obtinrent ainsi, que cette industrie soit protégée par des fortes taxes à l'importation); d'autre part, les producteurs de canne à sucre indépendants subordonnés du premier groupe; enfin, les journaliers ou saisonniers de sillon et de fabrique, travailleurs précaires sans voix.

7 Actuellement, d'après Vicente Sal ${ }^{3}$, la structure agro-sucrière est intégrée à environ 12 000 exploitations. Environ $80 \%$ des producteurs de canne à sucre indépendants possèdent des domaines de moins de 20 ha. Ils sont organisés dans l'Union de Producteurs de Canne Indépendants de Tucumán (UCIT), fondée en 1945. Les producteurs de canne à sucre peuvent être classés en 3 types :

8 - Les petits producteurs possèdent entre 5 et 10 hectares. Ils utilisent généralement une main-d'œuvre familiale. $10 \%$ de la récolte est faite manuellement, $30 \%$ est semimécanisée et $60 \%$ mécanisée. Parmi les problèmes auxquels ils doivent faire face, citons le manque de moyens financiers pour l'irrigation, pour l'achat des herbicides et pour assurer un transport rapide. En effet, les grandes distances que les chargements doivent parcourir jusqu'à la raffinerie de sucre, produisent des pertes de saccharose en 
raison du temps de parcours, qui se répercutent sur la qualité du sucre et bien sûr sur les prix. Soulignons un fait social d'importance : le paysan a peu de possibilités d'accumuler du capital car il ne possède ni la terre ni les moyens de production. Il doit compter sur la multi-activité de son groupe familial. On observe que les relations communautaires et d'organisation collective tendent à disparaître dans les communautés paysannes du NOA, ce qui participe à un appauvrissement de cette classe de producteurs de canne.

9 - Les moyens producteurs se différencient du groupe précédent par le fait, qu'ils sont totalement mécanisés; qu'ils diversifient leurs productions en cultivant du tabac et des légumes; qu'ils adhèrent à des coopératives. En effet, pendant les années 1960, les producteurs de canne à sucre ont commencé à s'organiser en coopératives de type commercial, dont la fonction était de vendre la canne à sucre de leurs membres, aux raffineries de sucre et dans le même temps, leur faciliter les crédits et autres bénéfices. Enfin, la main-d'œuvre des moyens producteurs n'est pas strictement familiale, au moment de la récolte (zafra) notamment, ils embauchent une main-d'œuvre saisonnière. La récolte manuelle de la canne à sucre demandait traditionnellement beaucoup de maind'œuvre qui arrivait des régions voisines et des provinces limitrophes. À partir des années 1970, on associe la récolte semi-mécanisée, puis la récolte intégralement mécanisée de la canne à sucre.

10 - En ce qui concerne les grands producteurs comme Los Cevilares, Paz, Frias Silva, ils s'organisent pour l'essentiel en 3 coopératives - Mayo, Campo Herrera et La Merced qui possèdent entre 80000 et 120000 sillons (surco).

11 À partir des années 1990, le poids des grandes exploitations s'accroît pour devenir des « méga-producteurs ».

12 En effet, Rofman (1999) décrit l'envolée de la productivité des actifs agricoles selon le niveau de mécanisation de la récolte : dans le système manuel, chaque actif récolte 1,5 tonne par jour (photo 1); dans un système semi-mécanisé, la productivité par individuest de 4 à 5 tonnes par jour ; avec la moissonneuse-lieuse intégrale (photo 2) on récolte 600 tonnes par jour! Les nouvelles technologies, et en particulier la mécanisation, sont à l'origine de l'affaiblissement voire de l'élimination économique, des plantations de canne à sucre de petite taille. Les petites exploitations sucrières ont diminué en nombre et en superficie. Soit elles disparaissent, soit elles s'orientent vers de nouvelles cultures. 
Photo $1:$ La récolte manuelle de la canne à sucre Hand harvesting of sugar cane

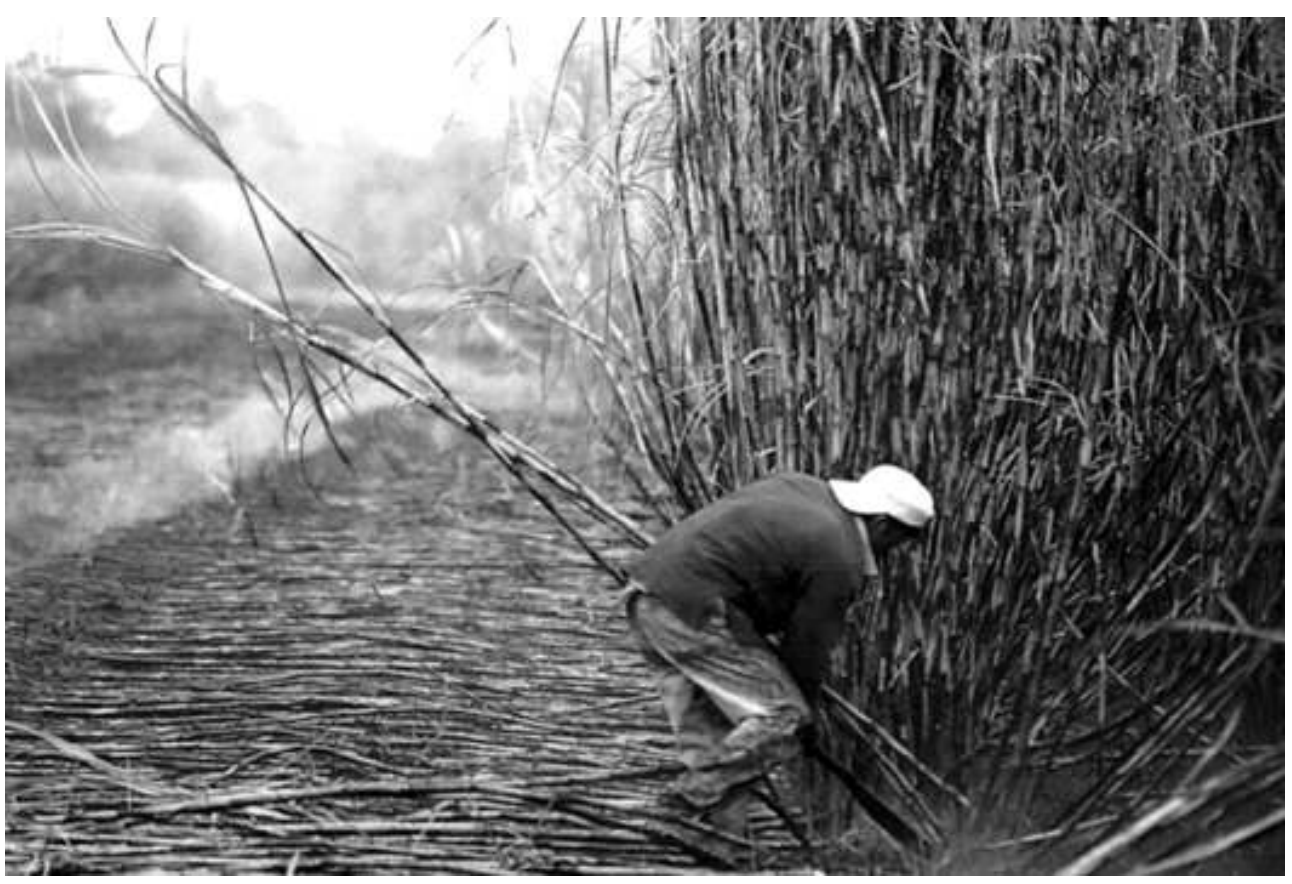

Photo 2 : La récolte mécanisée de la canne à sucre Mechanised harvesting of sugar cane

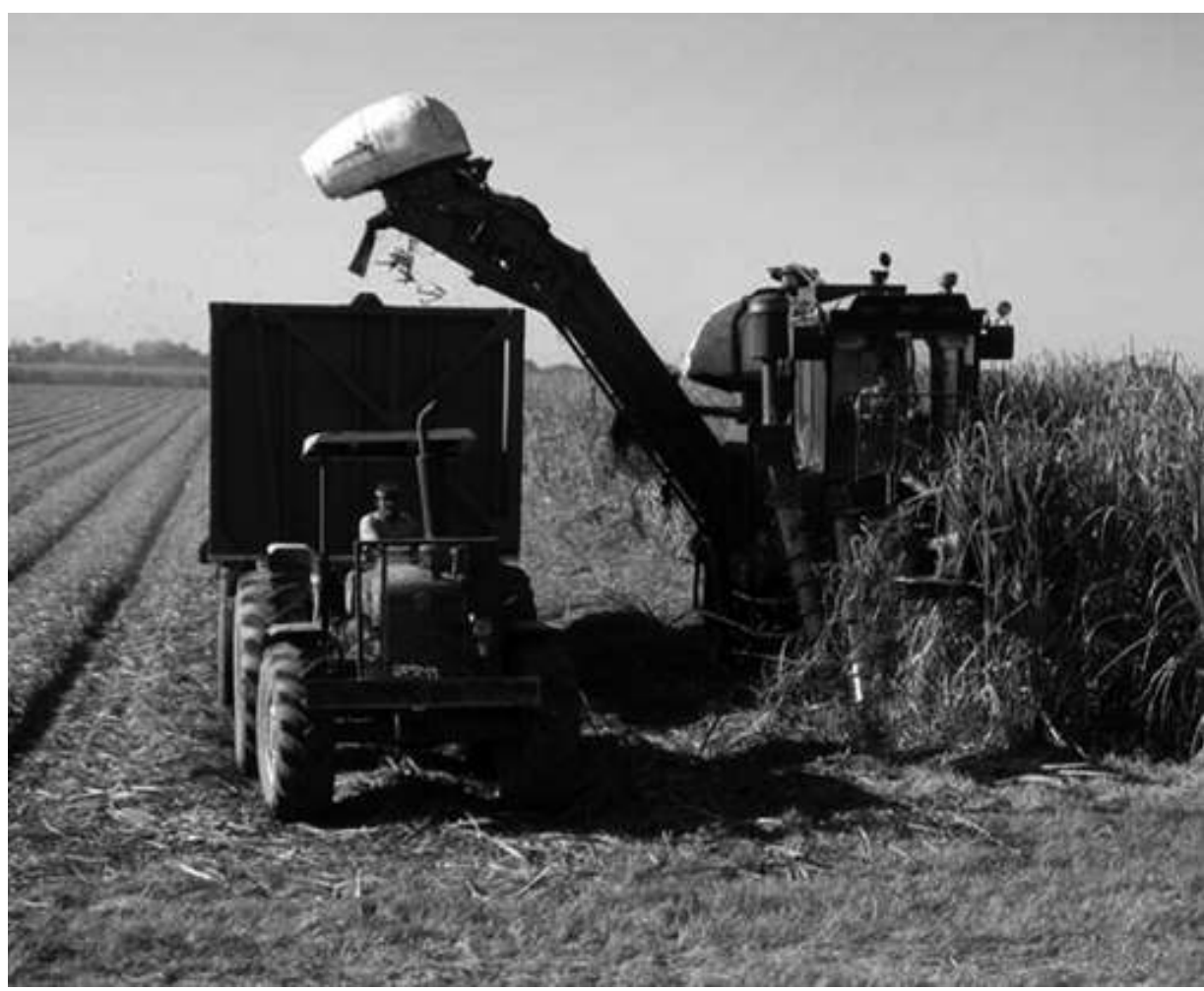

13 Profitant de ce contexte, des méga-producteurs acquièrent massivement les terres et achètent les petites raffineries en crise. Dans la province de Tucuman, ce sont 11 groupes qui possèdent des domaines sucriers qui varient entre 2000 et 10000 hectares, c'est-à-dire entre 500000 et 700000 tonnes de matière première et leurs raffineries. 
Parmi les plus importants on peut citer : José Minetti (2 raffineries), les Balcanes (2), ATANOR (3), Arcor SA (1), Konavle (1), Azucarera Argentina (1), Mijasi (1), SER (1), Azucarera Juan M. Terán (1), Las dulces del Norte (1), Complejo agroindustrial San Juan (1). Au total, ces 15 raffineries occupent environ 6710 travailleurs à l'époque de la récolte. Selon Rofman (2001), aujourd'hui, $10 \%$ des propriétaires concentrent les deux tiers du secteur sucrier de la province. Des conflits sociaux apparaissent comme conséquence de l'expansion de ce phénomène d'intégration capitaliste et de l'articulation de la communauté paysanne avec les raffineries dont ils sont dépendants (photo 3).

Photo 3 : La livraison des cannes à la raffinerie Delivering canes to the sugar plant

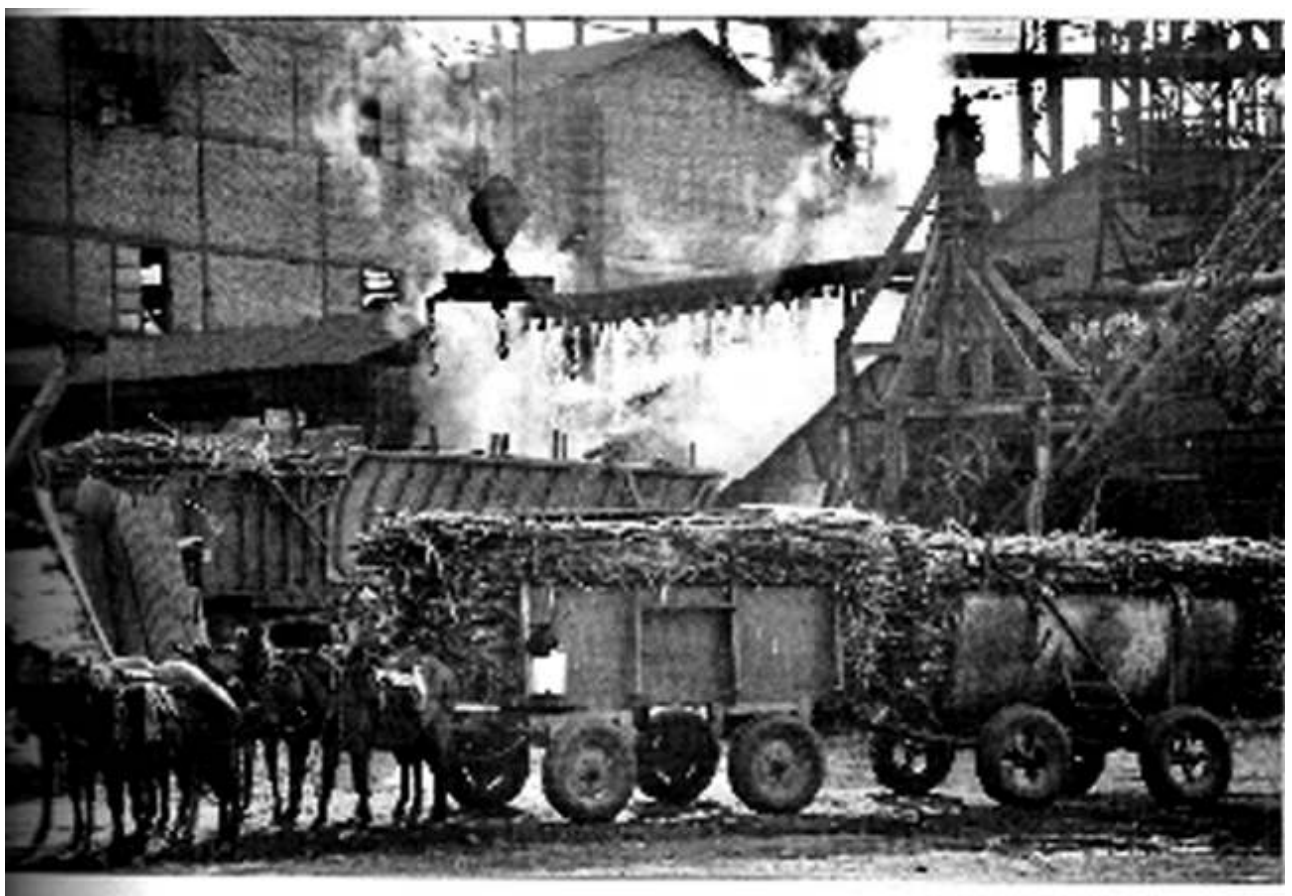

\section{Le rôle de l'État et ses effets sur les producteurs de canne}

Depuis la naissance de l'industrie sucrière, l'État a joué un rôle très actif. Premièrement il a créé un marché domestique protégé par des tarifs avantageux et attribué des subventions aux usines. Dans les années 1970, l'État a mené des rachats financiers d'usines endettées, pour éviter le chômage des ouvriers ; c'est-à-dire qu'il y avait une protection de l'État que les usines demandaient, invoquant la question sociale. Cependant, cela favorisait beaucoup plus les chefs des entreprises que les paysans.

La loi 19597 promulguée par l'État et approuvée en 1972, a créé le farinage : le producteur de canne livrait la raffinerie pour la fabrication du sucre, laquelle lui remettait $40 \%$ du sucre produit. Mais ce système ne réglementait pas les négociations pour la production d'alcool et de bagazo (matière première du papier) à partir de la canne. Vers les années 1980, avec la dérégulation néo-libérale, l'État n'intervient plus directement mais en qualité de surveillant. Pour ce faire, fut créée la Corporation des Producteurs de Canne Indépendants de Tucumán, qui centralise la gestion des producteurs. On voit alors naître le warrant, c'est-à-dire un certificat de dépôt du sucre 
dans une raffinerie. Grâce à ce certificat, les paysans peuvent obtenir des acomptes bancaires, dont la garantie serait l'existence prouvée de stocks de sucre en possession de l'industriel (propriété de celui-ci, des agriculteurs indépendants ou appartenant à une coopérative). Ce système existe toujours, mais il a donné des résultats variables. Parfois, il a généré des attitudes de corruption, il a relégué certains hors du marché, dans le chômage et la pauvreté.

Pendant les années 1990 où la crise argentine s'amplifie, il y eut une diminution du budget d'État ${ }^{4}$. Dans ce contexte de dérégulation, la loi 19.597 qui prenait en charge le coût du transport des cannes vers les raffineries, est restée sans effet. Ce processus a coïncidé avec la chute du prix du sucre provoquant la diminution de l'activité des usines.

17 L'adaptation des petits producteurs n'a pas été soutenue par le gouvernement provincial. Certains ont pu mécaniser leur système d'exploitation et converger vers les prix internationaux; d'autres ont dû chercher des secteurs plus dynamiques. Plus généralement, l'absence d'une politique d'État capable d'assurer aux producteurs, la sécurité juridique, la stabilité fiscale et la prévision quant aux résultats, constitue une des faiblesses du système sucrier.

\section{L'éthanol, le futur de la canne à sucre}

18 En ce qui concerne l'avenir de l'activité sucrière régionale, un optimisme, certes modéré, est de mise parmi les industriels sucriers dont les raffineries produisent $53 \%$ du sucre de Tucumán. Le point faible de la région est sa situation intérieure à distance des ports, aggravée par un système ferroviaire de livraisons absolument détérioré. Néanmoins, les raffineurs pensent que le secteur jouit d'une grande crédibilité puisqu'il répond de manière adéquate au marché interne et qu'il destine des excédents importants vers l'exportation. De plus, les conditions climatiques récentes favorables, l'adéquation mûrement réfléchie des investissements entre la campagne et la fabrique et les conditions intéressantes de coûts financiers dus à une offre bien adaptée de fonds pour les investissements, ont joué favorablement sur les prix internationaux. Une vision optimiste estime à 2 millions de tonnes de sucre la récolte 2010, ce qui permettrait de soutenir une partie du programme de l'éthanol à l'intérieur du programme de biocarburants impulsé par le gouvernement.

19 En effet, si l'on ajoute à la flambée des prix du pétrole, la crise énergétique que subit le pays, les biocarburants entrent de nouveau en vigueur comme source alternative d'énergie renouvelable. Coïncidant avec la préoccupation générale liée aux prix élevés de l'essence - et spécialement la diminution des réserves du brut américain à cause de la demande estivale - les trois entreprises automobiles les plus importantes des ÉtatsUnis (Général Motors, Ford, groupe Chrysler) ont annoncé conjointement, qu'elles doubleront d'ici 2010, la production de véhicules non dépendants des hydrocarbures. Le Président Bush a demandé à son gouvernement de commencer à agir pour atteindre l'objectif de réduire de $20 \%$ la consommation d'essence dans son pays, avec une projection sur 10 ans. De la même manière, il a assuré qu'il est important de réussir à diminuer la dépendance énergétique du pétrole provenant des régions politiquement instables par des mesures qui devront être mises en pratique vers la fin de 2008. Le gouvernement Bush considère la dépendance des États-Unis vis-à-vis du pétrole du Moyen-Orient comme une menace à la sécurité nationale. 

de litres d'éthanol dans la consommation, les émissions de bioxyde de carbone diminueraient de 3,5 millions de tonnes par an, l'équivalent de la mise hors circulation de 800000 véhicules à essence.

21 Voilà des perspectives qui pourraient encourager le développement de la filière sucrière sur d'autres bases que strictement alimentaires. La crise pétrolière serait-elle le pivot d'une amélioration économique du revenu de la province de Tucuman grâce à l'ouverture d'autres voies de valorisation de la production sucrière ? En effet, Tucumán peut jouer un rôle important grâce à son expérience dans la fabrication d'alcool. D'après une étude réalisée par Lannes ${ }^{5}$ et Pucci (2007) le fait de " couper » l'essence avec $5 \%$ de biocarburant à partir de 2010 en Argentine, permettrait d'accroître de $17 \%$ la production de canne à sucre, ce qui aboutirait à une croissance du $5 \%$ du PIB de Tucumán. Un autre avantage vient du fait que l'éthanol produit à partir de la canne à sucre, est plus rentable que celui obtenu à partir du maïs dans n'importe quelle partie du monde.

Ce programme en faveur du bio-éthanol remet au goût du jour le projet « Alconafta " (alcool-carburant) datant de 1981. À cette époque, Alconafta était décrit comme destiné aux régions "parents pauvres de l'intérieur du pays ", et où les avantages de ce carburant devaient encore essayer de convaincre les industriels et le gouvernement. Mais, selon Lannes et Pucci (2007), la tonne de sucre valait alors 900 US \$ sur le marché international et les discordes entre les États-Unis et l'Irak, ou le Venezuela, n'existaient pas encore.

\section{Le circuit productif du citron}

$\mathrm{Au} \mathrm{xx}^{\mathrm{e}}$ siècle, le secteur agricole argentin, et particulièrement celui de Tucumán, a subi des transformations influencées par le nouvel ordre économique mondial, ce qui a provoqué, depuis les années 1960, des changements dans la structure économique et sociale, occasionnant ainsi des extensions et des modifications de l'occupation agricole des terres, avec l'apparition du soja, du maïs, mais également de la production de citrons.

\section{Une production récente de contre-saison}

24 Cette activité citricole est exclusivement liée au marché externe, surtout des États-Unis et de l'Europe, en raison de la demande tant du fruit que de ses dérivés, dans la mesure où la production du Nord-Ouest argentin se fait à contre-saison par rapport à l'hémisphère nord.

Aujourd'hui, avec $90 \%$ de la production nationale, Tucumàn devient la première région à avoir industrialisé la production de citrons dans le monde. Il s'agit de la deuxième activité économique après la canne à sucre. Le citron se localise dans la province, le long du piémont, bande étroite qui s'étend de Burruyacu au nord, en passant par Tafí Viejo, Yerba Buena et Famaillá, jusqu'à la Cocha au sud, à une altitude moyenne de 300 à $500 \mathrm{~m}$, entre les limites d'isohyètes de 800 et $1500 \mathrm{~mm}$. Les ondulations topographiques des zones où prospère cette culture, apportent quelques avantages pédologiques comme l'élimination rapide des excès hydriques et l'atténuation des 
effets des très basses températures. On estime que $30 \%$ de la superficie cultivée dispose d'un système d'irrigation.

\section{Le fonctionnement du circuit}

Sur les 320000 hectares cultivés en canne à sucre dans les années 1960, 100000 hectares sont voués aujourd'hui au citrus ${ }^{6}$. L'essor de la production de citrus est sensible depuis 1966, la phase d'expansion la plus soutenue se situe entre 1988 et 1995 (Batista et Natera Rivas, 2001). Ces dernières années ont été marquées par une diminution de la surface cultivée à cause de la crise argentine. Néanmoins, cette tendance s'est interrompue en 2007 grâce à la reprise économique. Les citronniers " tucumanos " occupent environ 34000 hectares, avec une productivité supérieure à celle du reste du pays. Cela permet l'obtention d'un produit de bonne qualité à un prix compétitif.

Cette activité économique a donné lieu au développement d'une véritable filière. D'après l'Association du Citrus de Tucuman (ATC), les acteurs de la filière sont organisés dans une chaîne d'intégration verticale, formée par des producteurs agricoles indépendants, les emballeurs ou " packing » (40 au total) qui préparent le fruit pour sa commercialisation, les industries de conditionnement (8) et les exportateurs.

L'Association du Citrus de Tucuman (ATC) compte 350 associés. La surface des exploitations varie de 5 ha et à plus de 1000 ha. Les petites exploitations ont diminué fortement, alors que celles de plus de 20 hectares ont augmenté significativement. Les petits et les moyens producteurs produisent $40 \%$ des tonnages de citrons. Les grandes exploitations, qui exploitent $60 \%$ de la superficie cultivée en citrons, correspondent à un petit groupe de producteurs qui emploient de la technologie d'avant-garde.

Environ 40000 personnes participent à la récolte manuelle (photo 4). Ce service est généralement externalisé. Les ouvriers font la récolte par groupe et ils cueillent environ 100 musettes par jour. D'après les enquêtes réalisées, la récolte de citron pour l'exportation est mieux payée que celle destinée au marché interne. Huit usines de conditionnement dédiées au citron fonctionnent à Tucumán (photo 5), parmi lesquelles : Trapani, Citromax, Citrusvil, San-Miguel, Cota. Le traitement industriel du citron permet d'obtenir différents dérivés : jus concentré, zeste déshydraté et huile essentielle très demandée par la multinationale Coca-Cola, puisque c'est un des ingrédients de sa principale boisson. $70 \%$ de la production de la province sont destinés à l'exportation. Les pays de destination du citron de Tucumán, sont principalement la France, la Pologne, les Pays-Bas et l'ensemble des pays de l'UE (63\%), l'Est européen (30 \%), et par ailleurs la Russie et le Canada. Il faut souligner les mesures tendant à obtenir la réouverture du marché avec les États-Unis. Quant à la Chine, on devra attendre que son service phytosanitaire se prononce pour accorder le protocole d'exportation. 
Photo 4 : La récolte des citrons

Lemon harvest

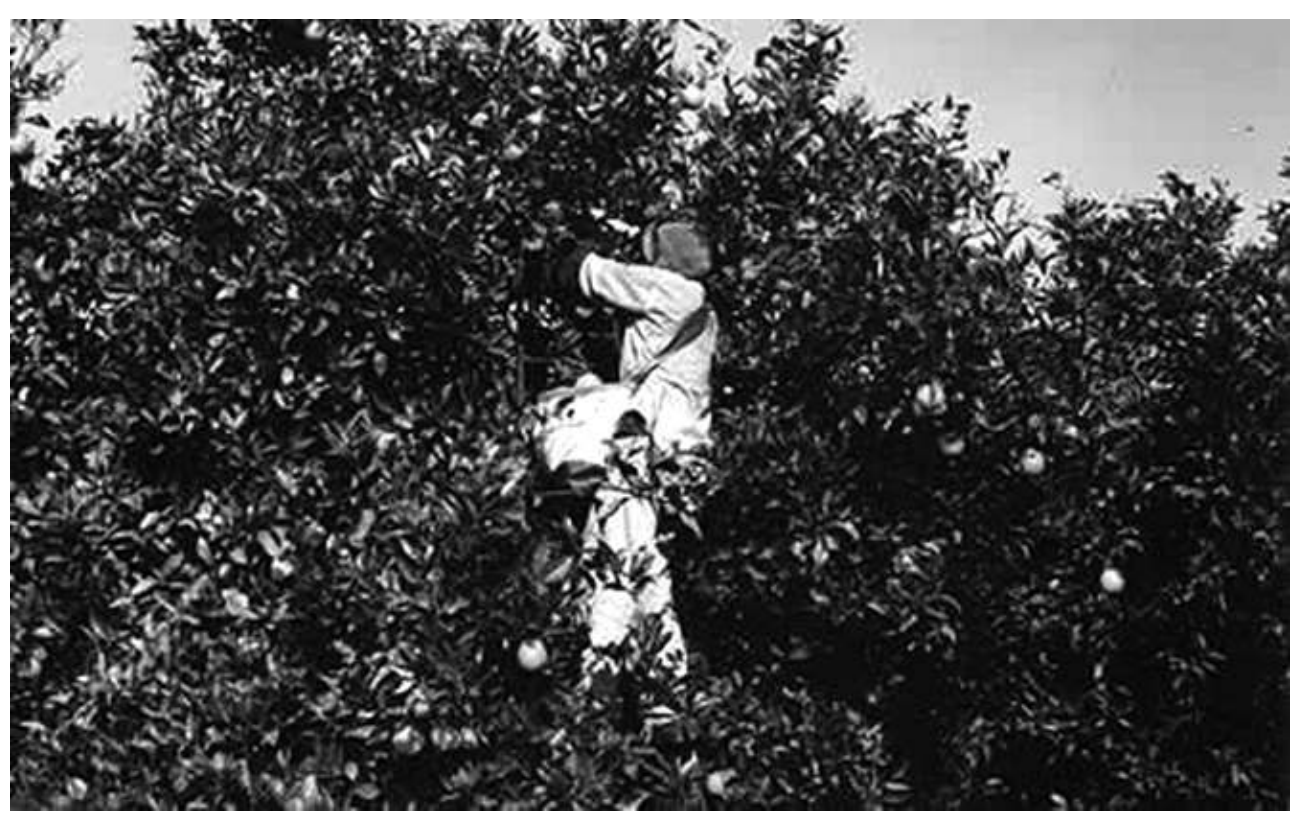

Photo 5 : Le conditionnement des citrons en usine Conditionning of lemon in the agro-unit

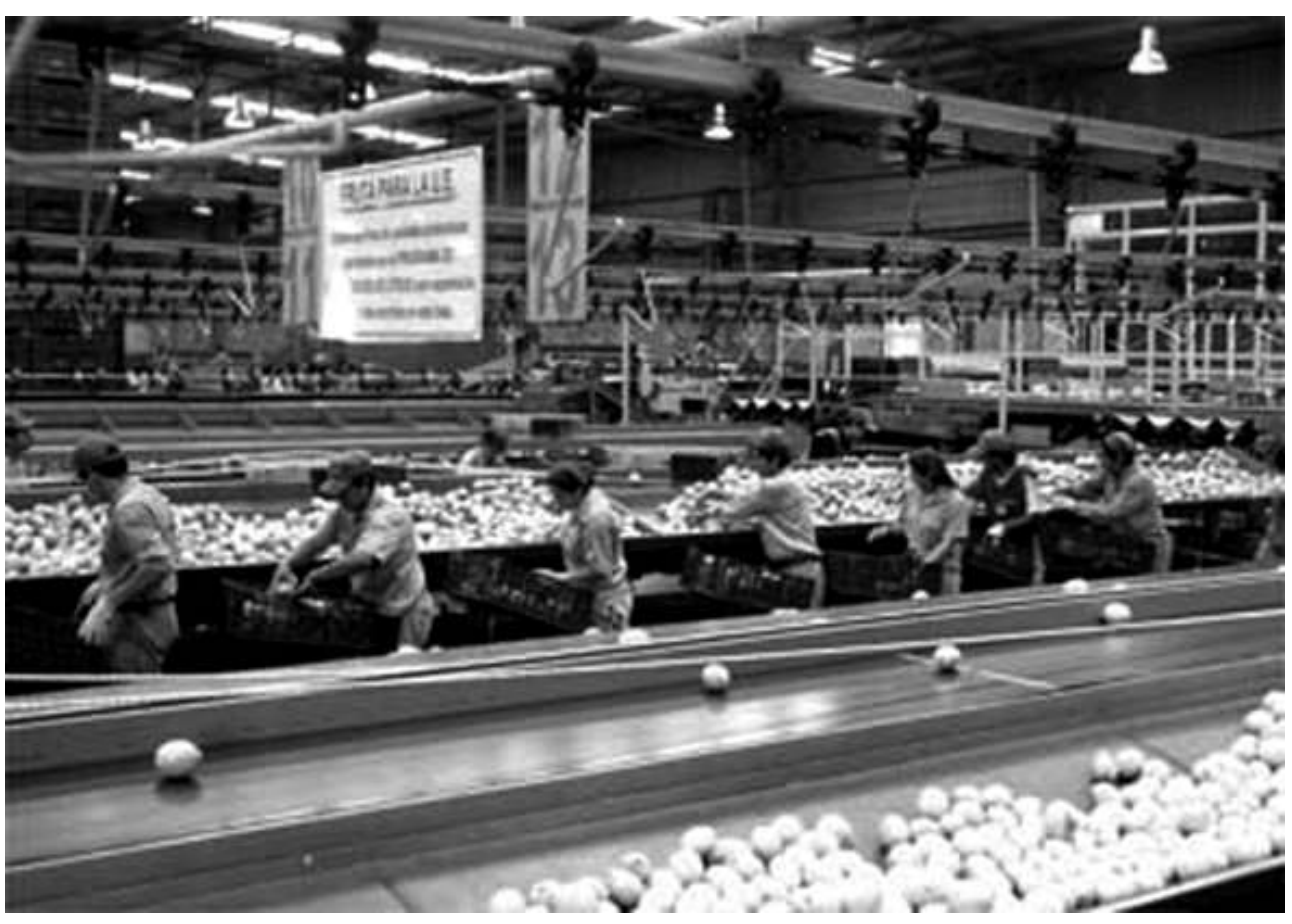

\section{Conclusion}

Les circuits productifs analysés ont occasionné des modifications du paysage agraire, ce qui a déterminé une extension de la frontière agricole vers l'est et le sud de la province du Tucumán. C'est l'ensemble des différents agents qui, grâce à leurs décisions, ont déterminé une croissance horizontale et verticale importante de l'économie de la 
province, avec d'importantes innovations technologiques conduisant à l'exploitation intégrale d'une ressource, l'agriculture, mais qui causent aussi de grands et rapides changements sur l'environnement. On attend de l'État des décisions politiques importantes pour éviter la spéculation et orienter ces filières vers une démarche nécessaire d'agriculture durable afin d'harmoniser la relation de la nature avec la société d'une part, et d'éviter le creusement d'inégalités socio-économiques profondes entre l'agro-industrie et la paysannerie un secteur historiquement opprimé, d'autre part.

\section{BIBLIOGRAPHIE}

BATISTA (A.), NATERA (R.), 2001. - Los cambios en el uso del suelo en el agro en Tucumán, contribución para problemas agrarios del noroeste argentino, Instituto de Estudios Geográficos, facult de Filosof y Letras, Université Nationale de Tucumán, Argentine, 52 p.

BATISTE (A. E.), 2002. - El complejo agroindustrial limonero y su articulación terrirorial en piedemento Tucumano (Argentina), Thèse,Département de Géographie, Université de Málaga, Málaga.

D’ARTERIO ORTíz (J. P.), RIVAS (A. I.), 2002. - Sobre el concepto del campesinado, Faculté de Philosophie et Lettres, Université Nationale de Tucumán, $4 \mathrm{p}$.

INSTITUT DE PLANIFICATION ET DÉVELOPPEMENT URBAIN, FACULTÉ D’ARCHITECTURE ET URBANISME, 1998. L'articulación de sistemas urbanos regionales NOA-Cuyo, Magna publications, p. 4-5.

INSTITUTO DE ESTUDIOS GEOGRAFICOS, 1997. - Problemas agrarios del noroeste argentino, Institut d'Études Géographiques, Faculté de Philosophie et Lettres de l'Université Nationale de Tucumán, San Miguel de Tucumán, Argentine.

LANNES (F.), PUCCI (C. A.), 2007. - La agroindustria azucarera y su influencia en el producto bruto interno de Tucumán, Informe, $20 \mathrm{p}$.

PUCCI (R.), 2004. - Historia de la destrucción de una provincia, Tucumán, Éditions del pago chico.

-, 1993. - « La elite azucarera tucumana y la formación del sector cañero entre 1880-1920 », Centro Editor de América Latina, Méjico, 13 p.

ROFMAN (A.), 1999. - Las economías regionales a fines del siglo XX. Los circuitos del petróleo, del carbón y del azúcar, Buenos-Aires, Ariel.

-, 2001. - Sistema socioeconómico y estructura regional en la Argentina, Buenos Aires, Edit. Amorrortu, $24 \mathrm{p}$.

RoSEnZVAIG (E.), 1997. - Historia social de Tucumán y del azúcar, Université Nationale de Tucumán, Argentine. 


\section{NOTES}

1. Le Président Juan Carlos Onganía a fermé 11 usines sucrières à Tucumán. Cela a été un " industricide » d'après l'historien Roberto Pucci (2004).

2. Le mot « agro-industrie » fait référence à la notion de circuit productif comme à l'ensemble des phases (agricole, industrielle, commerciale) et aux enchaînements successifs qui ont lieu entre elles, conduisant à un processus unique, qui va de l'obtention de la matière première initiale, jusqu'à la remise du produit élaboré au marché de consommation. De plus, l'idée de circuit aide à une meilleure compréhension des cadres régionaux du pays, parce que c'est un outil conceptuel et méthodologique d'explication des phénomènes et des processus qui s'y produisent entre des acteurs sociaux et des agents économiques. Le terme " agro-industrie » a commencé à être utilisé dans les années soixante-dix pour surmonter les anciennes divisions entre agricole et industriel, entre rural et urbain...

3. Il est le trésorier de l'Union de Producteurs de Canne à Sucre de Tucumán (UCIT) et PDG de la coopérative Villa de Leales.

4. Le gouvernement a lancé un réajustement financier aux provinces et a éliminé un financement aux banques officielles de l'intérieur du pays. À partir de 1992, la situation est devenue plus grave : Tucumán a du prendre en charge des frais de santé, d'assistance sociale et d'éducation. On a diminué les ressources de la coparticipation des provinces (Pucci,1993).

5. Federico Lannes est un ancien ministre de l'Économie et auteur du projet Alconafta.

6. Malgré une crise ces dernières années, la tendance à la réduction des surfaces en citrons s'est interrompue en 2007.

\section{RÉSUMÉS}

Le Nord-Ouest argentin (NOA) forme une partie de la région andine centrale de l'Amérique du Sud. Il est constitué par les provinces de Salta, Jujuy, Santiago del Estero et Tucumán. Dans cet article sont décrits deux circuits productifs de la région : celui de la canne à sucre et celui du citron. Cette analyse montre que, dans la conjoncture actuelle, une série de changements dans le domaine agricole bouleverse la province de Tucumán. Ainsi, la mécanisation de la récolte de la canne a enclenché un processus d'agrandissement des exploitations et de concentration de la filière au détriment des petites exploitations paysannes, renforcé par l'évolution vers la production de biocarburants. La filière du citron de contre-saison pour l'hémisphère nord participent également à ce remaniement au niveau territorial, économique et social de la province.

The NOA (Argentinian northwest) is part of the Central Andean region of South America. It comprises the provinces of Salta, Jujuy, Santiago del Estero, Catamarca and Tucumán. Two production patterns of the region are dealt with in this paper: one is bout sugar cane and the other about lemon. The the economic structure of production is highlighted because it is the core around which social issue are developping. It also allows to recognize the importance of the links with regional and national issues. This paper explains that in the current situation (from the late 20th century up to the present) a number of changes have been brought about in agriculture in the province of Tucumán which have had large territorial, economic and social implications. Mechanisation of cane harvesting has resulted in the enlargement of farms and in a 
concentration of the technical processes which has been harmfull for small farmers. The production of bio carb has also increased. The lemon harvest, being done during the cold season of the northern hemsiphere has also enhenced the effects of these territorial changes.

El NOA (Noroeste Argentino) es la parte de la región andina central de Sudamérica. Abarca las provincias de Salta, Jujuy, Santiago del Estero, Catamarca y Tucumán. En este trabajo se desarrollan dos circuitos productivos de la región: el de la caña de azúcar y el del limón. Se pone el acento en la estructura económica por ser el basamento sobre el que se desenvuelve la cuestión social y que a su vez permite reconocer el nexo externo de características regionales y nacionales. En el presente trabajo se parte de la hipótesis que en la actual coyuntura (fines del siglo XX hasta la actualidad) se han producido una serie de cambios en el agro que traen implicancias territoriales, económicas y sociales y particularmente en la provincia de Tucumán.

\section{INDEX}

Index géographique : Argentine, Tucumán

Keywords : agribusiness, economic agents, mega growers, production circuit, small medium and large growers, social actors, agricultural production

Palabras claves : actores sociales, agentes económicos, Agro-industrias, circuito productivo, megaproductores, mercado, pequeños medianos y grandes productores

Mots-clés : acteurs sociaux, agent économique, agro-industrie, bassin de production, filière, marché, production agricole, sucre

\section{AUTEUR}

\section{ELENA DEL VALLE CUETO}

Escuelas Experimentales de la Universidad Nacional de Tucumán 1553, rue Balcarce - San Miguel de Tucumán, Tucumán, Argentine, evc1101@hotmail.com 